\title{
Evaluation of the impact of viscosity, injection volume, and injection flow rate on subcutaneous injection tolerance
}

This article was published in the following Dove Press journal:

Medical Devices: Evidence and Research

II November 2015

Number of times this article has been viewed

\author{
Cecile Berteau' \\ Orchidée Filipe-Santos' \\ Tao Wang ${ }^{2}$ \\ Humberto E Rojas ${ }^{2}$ \\ Corinne Granger ${ }^{1}$ \\ Florence Schwarzenbach' \\ 'Becton-Dickinson Medical \\ Pharmaceutical Systems, Le Pont de \\ Claix, France; ${ }^{2}$ Eli Lilly and Company, \\ Indianapolis, IN, USA
}

Aim: The primary objective of this study was to evaluate the impact of fluid injection viscosity in combination with different injection volumes and flow rates on subcutaneous (SC) injection pain tolerance.

Methods: The study was a single-center, comparative, randomized, crossover, Phase I study in 24 healthy adults. Each participant received six injections in the abdomen area of either a 2 or $3 \mathrm{~mL}$ placebo solution, with three different fluid viscosities (1, 8-10, and 15-20 cP) combined with two different injection flow rates $(0.02$ and $0.3 \mathrm{~mL} / \mathrm{s})$. All injections were performed with $50 \mathrm{~mL}$ syringes and 27G, $6 \mathrm{~mm}$ needles. Perceived injection pain was assessed using a $100 \mathrm{~mm}$ visual analog scale (VAS) (0 mm/no pain, $100 \mathrm{~mm} /$ extreme pain). The location and depth of the injected fluid was assessed through 2D ultrasound echography images.

Results: Viscosity levels had significant impact on perceived injection pain $(P=0.0003)$. Specifically, less pain was associated with high viscosity (VAS $=12.6 \mathrm{~mm}$ ) than medium (VAS $=16.6 \mathrm{~mm}$ ) or low (VAS $=22.1 \mathrm{~mm})$ viscosities, with a significant difference between high and low viscosities $(P=0.0002)$. Target injection volume of 2 or $3 \mathrm{~mL}$ was demonstrated to have no significant impact on perceived injection pain $(P=0.89)$. Slow $(0.02 \mathrm{~mL} / \mathrm{s})$ or fast $(0.30 \mathrm{~mL} / \mathrm{s})$ injection rates also showed no significant impact on perceived pain during $\mathrm{SC}$ injection ( $P=0.79$ ). In $92 \%$ of injections, the injected fluid was located exclusively in SC tissue whereas the remaining injected fluids were found located in SC and/or intradermal layers.

Conclusion: The results of this study suggest that solutions of up to $3 \mathrm{~mL}$ and up to $15-20 \mathrm{cP}$ injected into the abdomen within 10 seconds are well tolerated without pain. High viscosity injections were shown to be the most tolerated, whereas injection volume and flow rates did not impact perceived pain.

Keywords: injection viscosity, injection speed, injection volume, subcutaneous, pain

\section{Introduction}

The use of large molecules, such as monoclonal antibodies, for treating a broad range of therapeutic areas including autoimmunity, osteoporosis, hypercholesterolemia, and cancer has significantly grown over the last decade. The proven medical benefit associated with the improvement of risk/effectiveness ratio of monoclonal antibodies in the treatment of advanced cancers, such as selected metastatic breast cancer with trastuzumab and selected metastatic colorectal cancer with cetuximab or bevacizumab, have positioned monoclonal antibodies as one of the major advancements in cancer treatment. ${ }^{1,2}$ Although intravenous (IV) route by infusion is the common delivery route for these drugs and is well accepted both by patients and health-care workers, ongoing research considers delivering monoclonal antibodies by subcutaneous (SC)
Correspondence: Florence

Schwarzenbach

Becton-Dickinson Medical Pharmaceutical Systems, II, rue Aristide Bergès,

F-38800 Le Pont de Claix, France

Tel +33476689407

Email florence_schwarzenbach@europe. bd.com 
injection as drug solution biodistribution to the targeted tissue seems to be improved. SC administration would be a preferred route of administration, offering patients the convenience of self-injection, reducing the need for onsite IV infusion treatment and, also lowering the risks of bloodstream infection and potential need for hospitalization. ${ }^{3-5}$ Indeed, some monoclonal antibodies are already approved for SC injection, ${ }^{3}$ such as trastuzumab in the treatment of HER2-positive breast cancer, ${ }^{6}$ omalizumab ${ }^{7}$ for patients with severe asthma, adalimumab ${ }^{8-10}$ for patients with autoimmune diseases, etanercept ${ }^{11}$ for the treatment of various inflammatory conditions, and denosumab ${ }^{12}$ for prevention of bone-related complications with solid tumors and bone involvement.

The development of a SC formulation of trastuzumab has shown that SC administration of monoclonal antibodies is a valid alternative to IV. .,13,14 $^{6}$

In some cases, these large molecules have very long half-lives, and the frequency of administration may not necessarily be related to their half-life, but rather dependent upon the dose volume that can be delivered in a single administration.

The viscosity of the formulation is one limiting factor when injecting a monoclonal antibody ${ }^{15-18}$ subcutaneously, particularly when the delivery device may require a fast injection rate. Today, most biologics are administered by $\mathrm{SC}$ injection, commonly in volumes not exceeding $1 \mathrm{~mL}^{19}$ and several recommendations limit the volume of SC injection to $1-2 \mathrm{~mL} .^{5}$ However, there is no evidence to support this limitation on volume ${ }^{19}$ and some recent studies suggest that SC injection volume up to $5 \mathrm{~mL}^{5,20}$ is well tolerated. To support this, a recent unpublished BD study with a bolus injector in development (BD Microinfusor [BD, Franklin Lakes, NJ, USA] clinical trial, study registration number AFSSAPS 2010-A00122-37) demonstrated that an SC injection of $2.9 \mathrm{~mL}$ viscous solution $(\sim 8 \mathrm{cP})$ with an electric pump was well accepted and tolerated at flow rates between 0.015 and $0.25 \mathrm{~mL} / \mathrm{s}$.

Devices with automatic functions represent an increasingly popular alternative to syringes for SC self-injection of parenterals. In theory, two different classes of devices with automatic functions could be used for administration: autoinjectors or bolus injectors. Autoinjectors must be maintained on the skin by the patient during injection, thus the injection duration is limited to approximately 10 seconds. Bolus injectors are fixed on the skin and enable the enduser to inject a relatively large volume of a viscous solution over a longer duration of time; however, patients may not necessarily accept this type of wearable device, preferring shorter injection times.

In a clinical trial recently published by Heise et al, ${ }^{21}$ the authors analyzed the role of multiple factors - injection volume (0.4-1.6 $\mathrm{mL})$; injection site (abdomen or thigh); injection rate $(0.15$ or $0.45 \mathrm{~mL} / \mathrm{s})$ - in the tolerance of an SC injection of $0.9 \%$ saline solution. A major factor influencing subject tolerance assessed using a $100 \mathrm{~mm}$ visual analog scale (VAS) was pain at the injection site (both statistically and clinically significant favoring abdomen over thigh). Pain intensity scores were statistically higher with larger volumes, but it was no different than scores for abdomen $\mathrm{SC}$ injections. Interestingly, injection rate did not play any role in subject's tolerance ${ }^{21}$ as was seen in previous studies performed in house.

However, based on available data today (published and unpublished), it has not been possible to determine the maximum volume and viscosity that can be delivered during a 10-15-second injection with an autoinjector and the impact of drug viscosity on subject tolerance. This study analyzes the role of flow rate (injection speed) and drug viscosity on the tolerance of 2 and $3 \mathrm{~mL}$ SC injections, which corresponds to the injection volume of several monoclonal antibodies in development.

\section{Methods}

\section{Study design and participants}

The goal of this study was to evaluate the impact of fluid viscosity, speed of injection (flow rate), and injection volume in SC injection tolerance, defined by pain, in healthy subjects. The secondary goals were to evaluate the impact of the same parameters on skin reactivity at injection site and on accuracy of fluid depot location.

This study was a comparative, randomized, singleblinded, crossover Phase I study in healthy volunteers. All volunteers provided written informed consent to participate in this study. Subjects were blinded regarding the viscosity, flow rate, and volume of the injections. It was conducted in accordance with the Declaration of Helsinki 1964, revised in 2008, and the guidelines for Good Clinical Practices. The protocol was approved by the Ethics Committee: CPP Sud-Est III (Lyon, France) and French Health Agency (trial registration number 2013-A00126-39).

\section{Participants}

Sixty subjects were screened to enroll a total of 24 healthy subjects, 12 males and 12 females, between 35 and 50 years old with no chronic diseases or concomitant treatments that would 
interfere with pain assessment, with no abnormal skin conditions in abdomen that would prevent site assessment at the time of screening, and with a body mass index (BMI) $\geq 18.5 \mathrm{~kg} / \mathrm{m}^{2}$. No major protocol violation was recorded.

\section{Study design}

Each participant received six SC injections of normal $0.9 \%$ saline solutions with different concentrations of nonanimal hyaluronic acid in order to cover viscosity ranges of low (1 cP), medium (8-10 cP) to high $(15-20 \mathrm{cP})$. The solutions were injected at two different flow rates $(0.020$ and $0.30 \mathrm{~mL} / \mathrm{s}$ ), with two different target volumes ( 2 and $3 \mathrm{~mL}$ ). As it was not possible to administer 12 injections per subject
$(2 \times 3 \times 2)$ and, in order to cover all possible combinations of viscosity and injection flow rates $(2 \times 3)$ for a given targeted volume, each subject received six injections of the same target volume, either 2 or $3 \mathrm{~mL}$. Investigation lasted 7 hours ( 3 hours in the morning, 1 hour break, and 3 hours in the afternoon), including six injections with a 30 -minute pause after each injection (Figure 1).

\section{Sample size}

The number of required participants was determined by a crossover multifactorial design with three factors qualifying the injection: three viscosities, two flow rates, and two volumes. Although the sample size was not selected

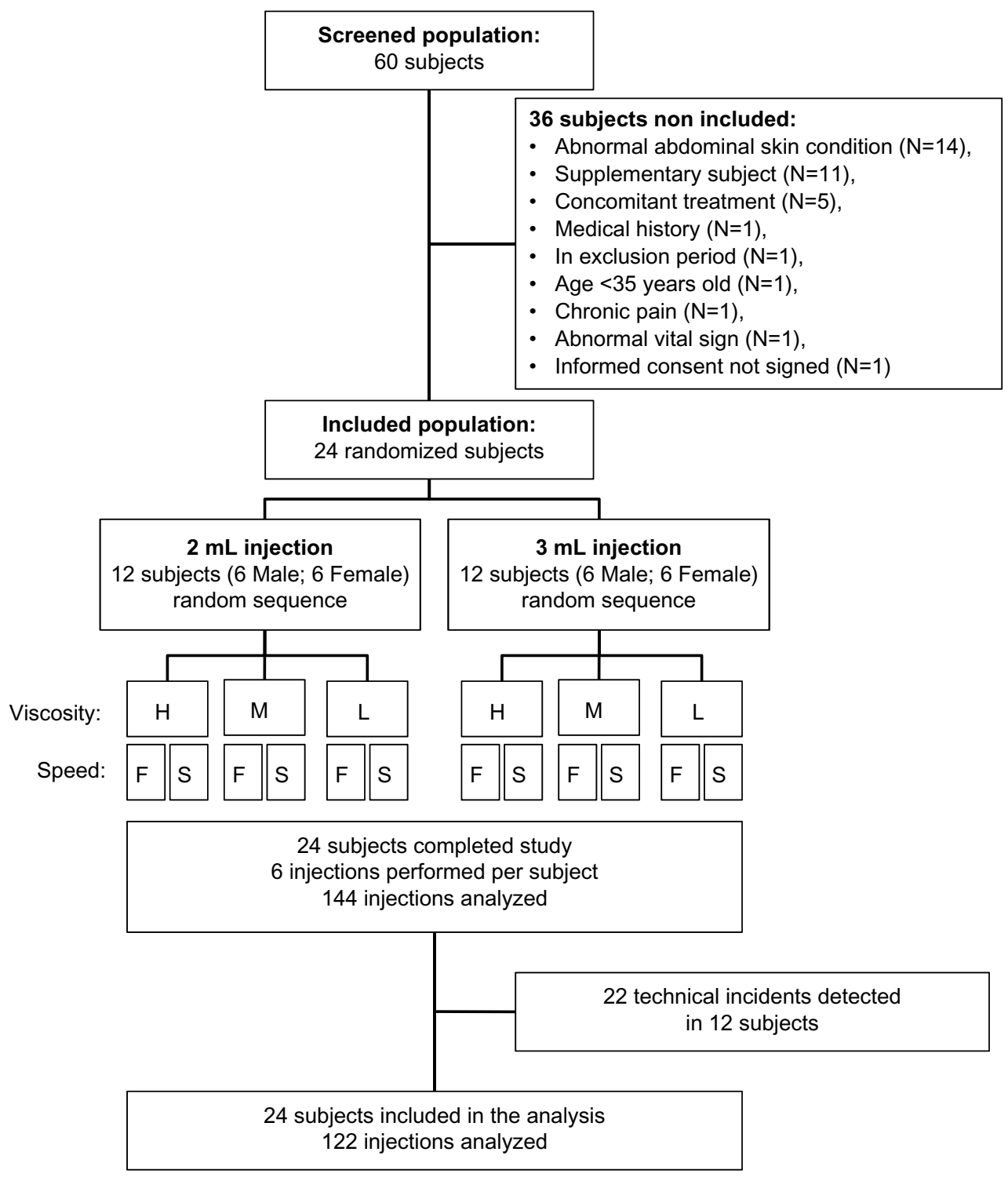

Figure I Study subjects enrollment.

Notes: Viscosity: H, high (I5-20 cP); M, medium (8-10 cP); L, low (I cP). Speed: F, fast $0.30 \mathrm{~mL} / \mathrm{s}$; S, slow $0.20 \mathrm{~mL} / \mathrm{s}$. 
based on statistical power consideration, the crossover design greatly increased the power of statistical comparisons when compared with a conventional simple factorial design. Specifically, each combination of the three factors was repeated 12 times, which gave 144 injections in total.

\section{Randomization}

Six males and six females were randomly allocated to either the 2 or $3 \mathrm{~mL}$ injection group. Within each of these groups, the order of injection was selected randomly to balance the three levels of viscosity and the two injection rates as well as sex (Figure 1) and injection site location (Figure 2).

\section{Procedure}

\section{Delivery system}

All injections were performed with the same model of electric pump. The injection procedure is depicted in Figure 2. Injections were performed using a delivery system composed of marketed devices. A $50 \mathrm{~mL}$ BD Plastipak ${ }^{\mathrm{TM}}$ (Becton Dickinson, San Agustin, Spain) syringe was connected to an electric pump (Fresenius Agilia MC; Fresenius Vial, Brezins, France) to enable controlled speed of injection. The Luer Lock Syringe was linked to a $27 \mathrm{G}$ needle (Microinjection needle $0.4 \times 6 \mathrm{~mm}$ thin wall Mesalyse ${ }^{\circledR}$; Puiseux le Hauberger, France) with an extension line (BD Connecta ${ }^{\mathrm{TM}}$; Becton Dickinson). To mimic an autoinjector or a bolus injector, the needle was introduced perpendicularly to the skin. A foam pad was used to maintain this position during the entire duration of the injection and to ensure a standardization of the injection depth of $6 \mathrm{~mm}$. The delivery schema is depicted in Figure 2A. In order to avoid bias due to difference of skin sensitivity, the abdomen was divided in six different parts, (Figure 2) allocated by randomization for each injection, and the sensitive area around the navel was avoided by keeping at least a distance of $2 \mathrm{~cm}$ between the navel and point of injection.

\section{Injectable solutions}

Three solutions were prepared from a sterile normal $(0.9 \%)$ sodium chloride $(\mathrm{NaCl})$ solution, corresponding to the low viscosity $1 \mathrm{cP}$ solution, then adding nonanimal origin hyaluronic acid (Vivacy Laboratories, Paris, France) to the $\mathrm{NaCl} 0.9 \%$ solution to reach the target viscosity of the solution: 8-10 cP for the medium- and 15-20 cP for the high-viscosity solution.

\section{Assessments conducted during the study Pain evaluation}

Participants were asked to evaluate their perceived injection pain through a $100 \mathrm{~mm}$ VAS $(0 \mathrm{~mm} / \mathrm{no}$ pain, $100 \mathrm{~mm} /$ extreme pain), immediately after needle insertion and immediately after injection completion.

\section{Actual volume injected}

The volume injected was derived from subtracting the residual (postinjection) volume in the syringe from the preinjection volume (calculated by the weight difference of the syringe before and after the injection) minus the leakage volume (calculated by the weight difference of a wicking spear collecting the leaked fluid).

\section{Skin reactivity}

Local injection reactivity was assessed by collecting signs of bleeding, erythema, swelling, and hematoma formation immediately after the completion of the injection and approximately 15 minutes ( \pm 5 minutes) after injection. Assessment was made according to a $0-3$ the scale as described in ISO

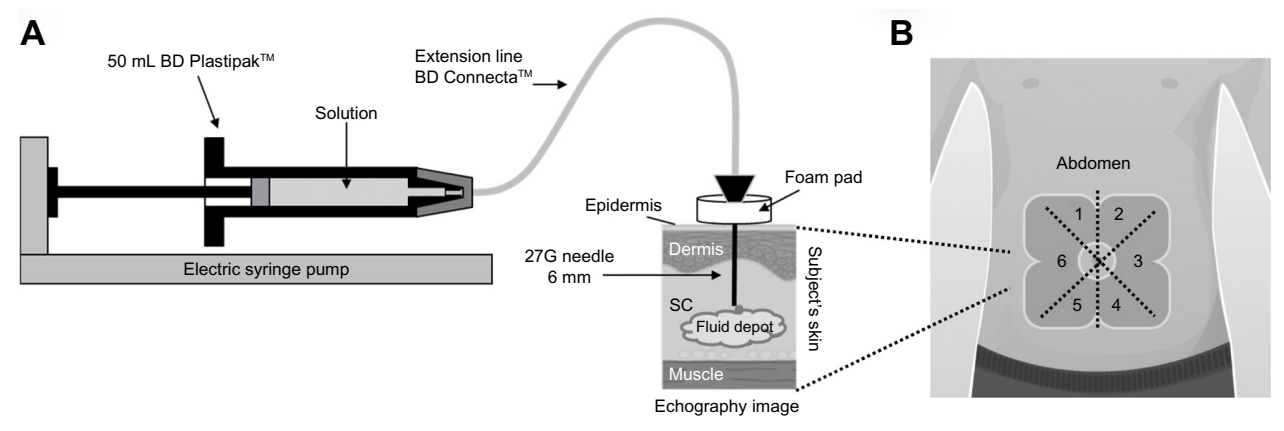

Figure 2 Injection procedure and injection site location.

Notes (A) Injection procedure. The delivery system was composed of plastic syringe $50 \mathrm{~mL}$ (BD Plastipak ${ }^{\mathrm{TM}}$ ) connected to one electric pump (Agilia ${ }^{\circledR}$ Fresenius) and linked to a $27 \mathrm{G} 6 \mathrm{~mm}$ needle (Mesalyse ${ }^{\circledR}$ Microinjection needle) through an extension line (BD Connecta ${ }^{\mathrm{TM}}$ ). The needle was introduced perpendicularly to the skin. A foam pad was used to maintain this position during the entire duration of the injection. (B) Injection site location. Each subject received six injections in the abdomen area, divided into two sessions, one in the morning $(I, 3,5)$, the other in the afternoon $(2,4,6)$.

Abbreviation: SC, subcutaneous. 
10993-10 standard: $0=$ no skin reactivity; $1=$ mild (the subject is aware of the sign/symptom, but finds it easily tolerated), $2=$ moderate (the subject has discomfort enough to cause interference with usual activities); $3=$ severe skin reactivity (the subject is incapacitated and unable to work or participate in many or all usual activities).

\section{Fluid location and depth}

The location of the injected fluid in the body tissue layers was assessed by echography (two-dimensional ultrasound method in B mode, $25 \mathrm{MHz}$ probe or more, high resolution) of the injection site, performed before (as control) and immediately after each of the six injections (Figure 2B). The radiologist was blinded to all injection parameters (volume, viscosity, and injection flow rate). The location and depth of the injected fluid in the body tissue layers were categorized as being exclusively $\mathrm{SC}$ or not. Injection depth was defined as the distance between the skin surface and the bottom of the fluid.

\section{Statistical analyses}

Perceived injection pain upon completion of injection was analyzed using a mixed model for repeated measures with the following fixed effects: injection viscosity, injection volume, injection flow rate, session (morning or afternoon), sequence of administration, and sex. The subject was also included in the model to account for the multiple measures made on each subject. Perceived pain after needle insertion was included in the model as a covariate. Other quantitative parameters were analyzed according to the same model without the covariate. Because conditions to use a logistic regression model were not met, qualitative criteria were analyzed by nonparametric methods (ie, generalized Cochran-Mantel-Haenszel or Kruskall-Wallis test) for each of the following factors: viscosity, volume, and injection rate, separately.

All analyses were performed using the statistics software package SAS version 9.1 (SAS Institute Inc., Cary, NC, USA) by Eurofins OPTIMED Clinical Research (Gières, France). For all statistical tests, $P$-values lower than 0.05 were considered statistically significant.

All participants enrolled in the study completed the study and were included in the analysis population. The analysis was performed with all 144 performed injections.

Among the 24 subjects, 12 experienced at least one injection system occlusion during the study, for a total of 22 recorded occlusions (Figure 1). Technical incidents appeared only with the $3 \mathrm{~mL}$ injections at the fast flow rate. These technical incidents appeared in $18.2 \%, 27.3 \%$, and
$50 \%$ of injections of the low-, medium-, and high-viscosity injections, respectively. In all the cases except one, the occlusion occurred after most of the targeted volume had been injected. The percentage of solution actually injected versus the targeted volume was similar across the 2 and $3 \mathrm{~mL}$ injections, as well as for the slow and fast flow rate injections.

In order to evaluate the impact of injection system occlusions on the study results, a complementary analysis was conducted including only the 122 nonoccluded injections, to compare if the exclusion of the 22 injections with occlusion had any impact on the results observed for the overall 144 planned injections.

\section{Results \\ Demographic and clinical characteristics of participants}

Equal number of females and males (12 in each sex) were enrolled in this study (Figure 1), with ages ranging from 35 to 50 years. The weight and height ranged from 48.9 to $99.7 \mathrm{~kg}$ and 150 to $195 \mathrm{~cm}$, respectively. The BMI ranged from 20.1 to $28.4 \mathrm{~kg} / \mathrm{m}^{2}$. General demographic data are summarized in Table 1. No significant differences in demographic parameters were found between the subjects receiving either 2 or $3 \mathrm{~mL}$ injections. No history of chronic disease or abnormal abdominal skin condition was recorded for enrolled subjects; they were all assessed as healthy. Each participant received six SC injections in the abdomen as depicted in Figure 2.

\section{Injection viscosity, volume, and flow rate effect on perceived pain}

Perceived pain was evaluated for each of the 144 injections immediately after needle insertion and at the end of injection. The mean (median) values of VAS pain were 12.0 (6.0) and 17.1 (9.5) $\mathrm{mm}$ after needle insertion and at the end of injection, respectively (Table 2 and Figure 3 ).

Table I Demographic characteristics (mean \pm SD) of the study participants

\begin{tabular}{llll}
\hline $\begin{array}{l}\text { Demographic } \\
\text { data }\end{array}$ & Mean \pm SD & & \\
\cline { 2 - 4 } & Male $(\mathbf{N}=\mathbf{I 2})$ & Female $(\mathbf{N}=\mathbf{I 2})$ & Total $(\mathbf{N}=\mathbf{2 4})$ \\
\hline Age (years) & $42.9 \pm 3.6$ & $39.3 \pm 3.3$ & $4 I . I \pm 3.9$ \\
Height $(\mathrm{cm})$ & $180.7 \pm 7.3$ & $164.0 \pm 6.6$ & $172.4 \pm 10.9$ \\
Weight $(\mathrm{kg})$ & $78.6 \pm 11.7$ & $61.3 \pm 6.7$ & $69.9 \pm 12.8$ \\
BMI $\left(\mathrm{kg} / \mathrm{m}^{2}\right)$ & $23.9 \pm 2.3$ & $22.8 \pm 2.7$ & $23.4 \pm 2.5$ \\
\hline
\end{tabular}

Note: 24 healthy individuals were enrolled in the study. Abbreviations: SD, standard deviation; BMI, body mass index. 


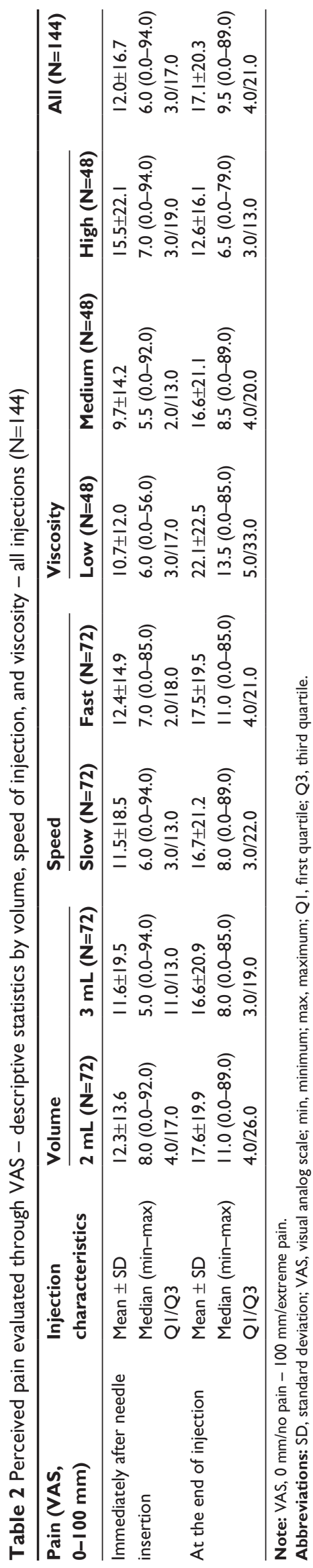

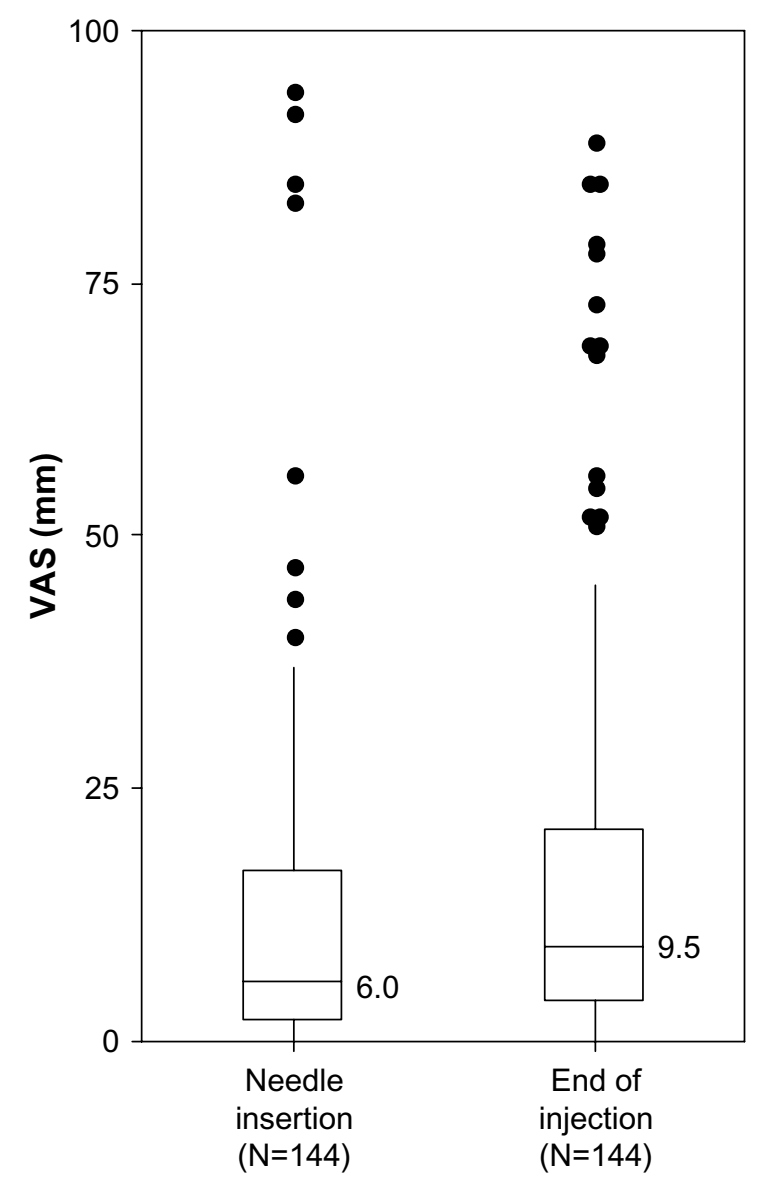

Figure 3 Perceived injection pain for all 144 injections at needle insertion and after injection completion.

Note: VAS was used to assess pain and goes from $0 \mathrm{~mm} / \mathrm{no}$ pain to $100 \mathrm{~mm} /$ extreme pain; medians are indicated.

Abbreviation: VAS, visual analog scale.

Mean (median) VAS pain scores at the end of injection were 22.1 (13.5), 16.6 (8.5), and 12.6 (6.5) $\mathrm{mm}$ for the low-, medium-, and high-viscosity groups. There were statistically significant differences among the three groups of injection viscosity $(P=0.0003)$ (Table 2 and Figure 4). Among the four multiplicity adjusted pairwise comparisons, the $10.7 \mathrm{~mm}$ difference of least square means between the low- and high-viscosity group was the only one that was significant $(P=0.0002)$. Although these results were not statistically significant when excluding the 12 injections with obstruction (by the analysis performed on the 122 complete injections), the trend in viscosity effect on perceived pain was maintained $(P=0.08)$ (Figure $\mathrm{S} 1$ ).

At the end of injection, the mean pain difference between the $2 \mathrm{~mL}$ and the $3 \mathrm{~mL}$ groups was not statistically significant $(P=0.89)$. Similarly, there was no statistically significant difference between the slow $(0.02 \mathrm{~mL} / \mathrm{s})$ and fast $(0.30 \mathrm{~mL} / \mathrm{s})$ flow rates $(P=0.79)$. The subsequent analysis conducted with the 122 complete injections confirmed these findings. 


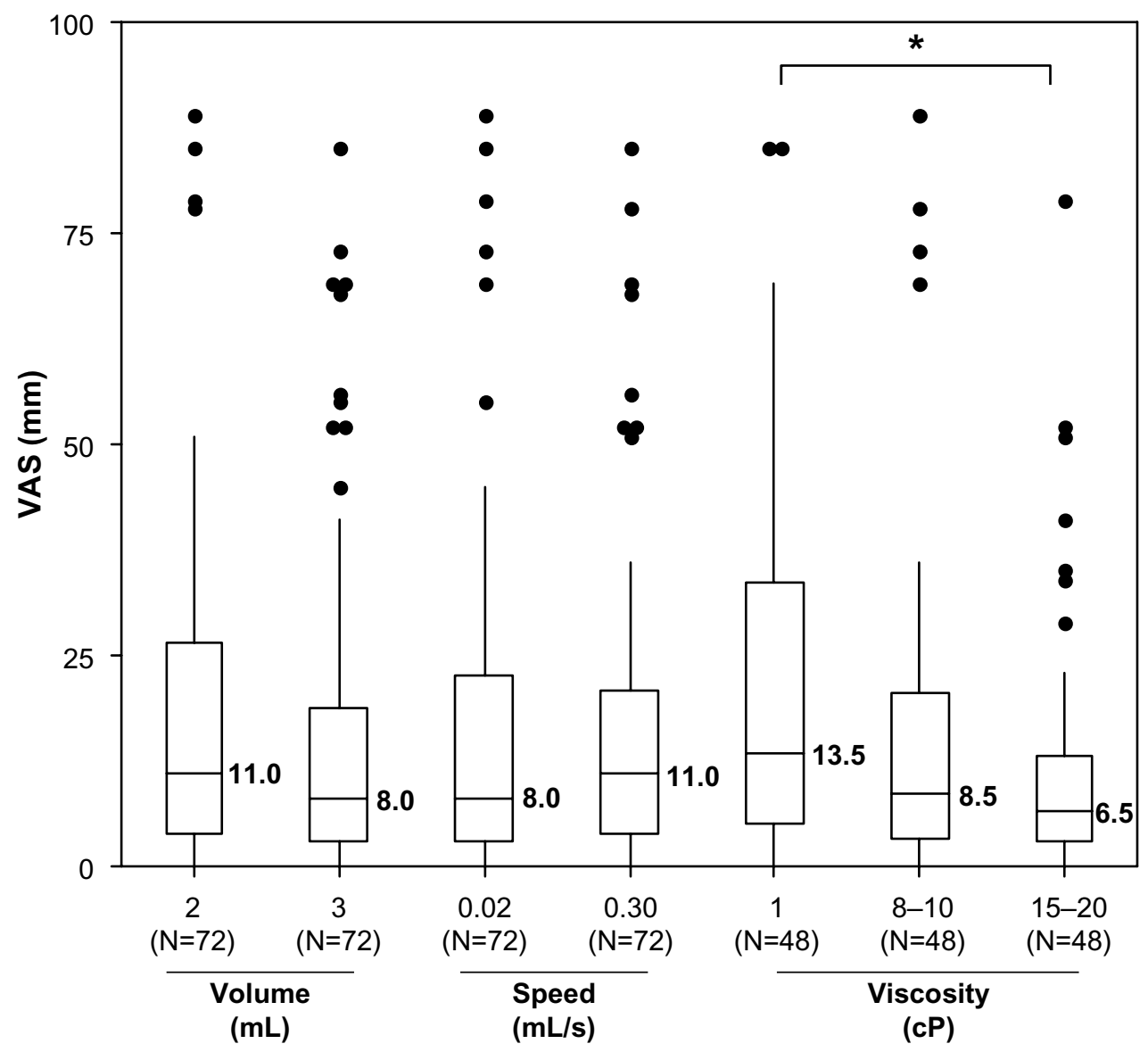

Figure 4 Perceived injection pain after the end of the 144 injections, per group of volume, speed of injection, and viscosity.

Notes: VAS was used to assess pain and goes from $0 \mathrm{~mm} / \mathrm{no}$ pain to $100 \mathrm{~mm} /$ extreme pain; medians are indicated. A significant difference is observed across viscosity injections $(* P=0.0003)$.

Abbreviation: VAS, visual analog scale.

There was no significant impact in perceived pain after SC injection of up to $3 \mathrm{~mL}$ volume and up to a $0.30 \mathrm{~mL} / \mathrm{s}$ flow rate in the abdomen (Figure 4).

Overall, high-viscosity injections were the most tolerated, whereas no impact on perceived pain was detected when varying injection volumes and flow rates.

\section{Injected fluid location}

In order to assess if the injected fluid was located in the SC space throughout the different conditions of the injections, echography images for all participants were taken at the site of injection before and after each injection to assess fluid location and depth (Figure 5A). Echography images showed that the injected solution was exclusively in the SC tissue in $92 \%$ of cases, this ratio did not change significantly across injection configurations (Figure 5B). The proportions of injections located exclusively in the SC location was significantly higher with $3 \mathrm{~mL}$ injections $(P=0.0213)$; it was also the case with increasing viscosity solutions, but the difference was not significant $(P=0.14)$ (Figure 5B). Twelve (8\%) of the 144 injections were not exclusively SC: nine were in intradermal and SC, and three were exclusively intradermal. Four of these 12 injections were found in the same subject (in the $2 \mathrm{~mL}$ group), who had a BMI of 22.1 $\mathrm{kg} / \mathrm{m}^{2}$. They were observed most frequently in males (nine cases) and in the $2 \mathrm{~mL}$ group (ten cases). Neither viscosity $(P=0.34)$ nor flow rate $(P=0.94)$ had an effect on fluid injection location. No pattern in injection and/or subject characteristics related to the intradermal location of fluid could be identified.

\section{Skin tolerance}

Immediately after injection the occurrence of erythema, swelling/induration, and local bleeding was respectively seen in $40 \%, 38 \%$, and $40 \%$ of the injection sites. Fifteen minutes after the occurrence of these symptoms, the values changed to $72 \%, 15 \%$, and $9 \%$, respectively. No hematomas were observed immediately after injection, but one mild case was 

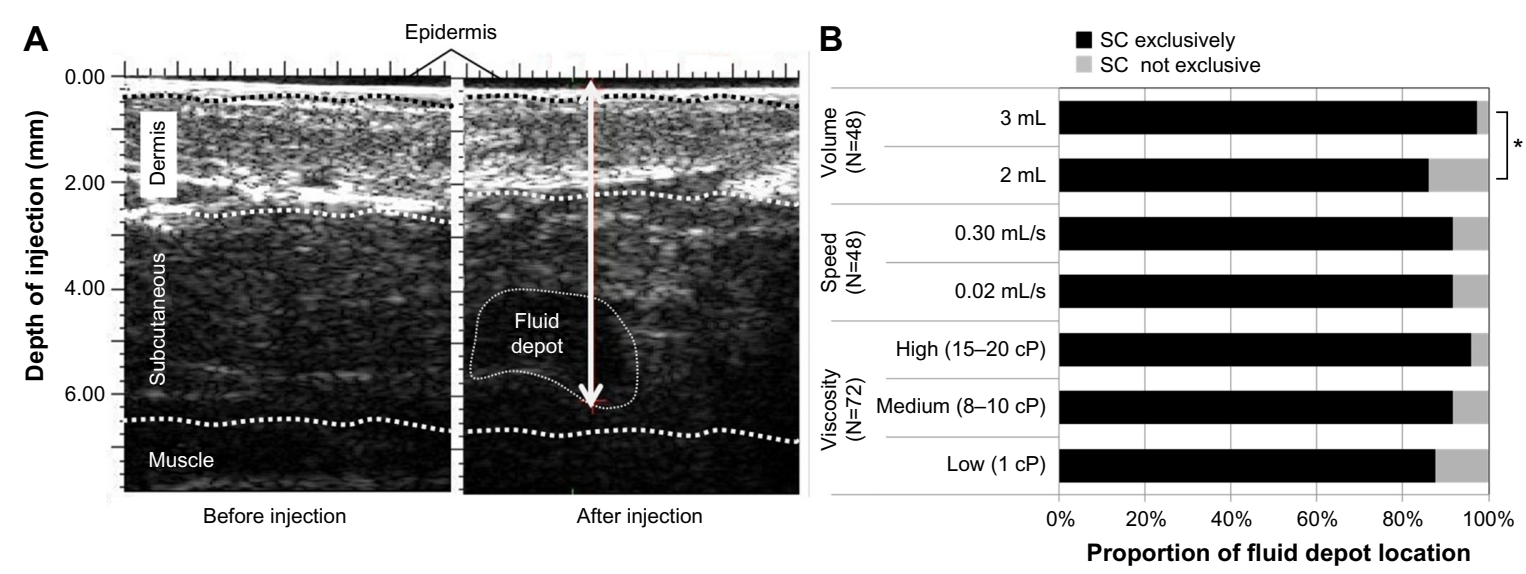

Figure 5 Injected fluid location after injection $(\mathrm{N}=\mid 44)$.

Notes: (A) Example of typical 2D-ultrasound B-mode echography of the injection site in the abdomen, before (left) and after (right) injection in the same subject. The white arrow measures the injection depth. (B) Fluid location evaluated by echography images, percentage of fluid located exclusively in $S C$ tissue. Significant difference $(* P=0.02 \mathrm{I} 3$ ) between 2 and $3 \mathrm{~mL}$ injections: fluid located exclusively in SC tissue more frequently with $3 \mathrm{~mL}$ injections.

Abbreviation: SC, subcutaneous; 2D, two-dimensional.

observed 15 minutes after the injection in a patient receiving $2 \mathrm{~mL}$ of medium viscosity at a fast flow rate.

No severe reactions were recorded for local bleeding, erythema, and hematoma. In 2 of the 144 injections (1.4\%), swelling/induration was considered severe immediately after the injection and became moderate 15 minutes later. Both were observed in the $3 \mathrm{~mL}$ group with the fast rate, resulting in a swelling/induration rate of $16.6 \%$ for this subgroup; this difference is significant $(P=0.0158)$. One other severe swelling/ induration was recorded 15 minutes after a $3 \mathrm{~mL}$ slow-rate low-viscosity injection. The echography image of that injection indicated that the fluid was delivered exclusively in dermis.

Overall, with the exception of the $3 \mathrm{~mL}$ injected at $0.3 \mathrm{~mL} / \mathrm{s}$, skin tolerance appears to be acceptable in the different tested injection conditions, even for high-viscosity injections.

\section{Actual volume injected}

Leakage was negligible (less than $5 \%$ of the injected volume) with an average of $3.8 \mu \mathrm{L}$ (and standard deviation of $9.2 \mu \mathrm{L}$ ). It was observed in $50 \%$ of injections, and no difference was observed among the various types of injections.

Evaluation of the delivery system accuracy was performed by estimating the actual injected volume as described in the "Methods" section. Overall, the real injected volume was $83 \%$ of the targeted one and appeared to be homogeneously distributed across the different volume and flow rate conditions.

It was significantly lower $(P<0.001)$ with the highviscosity solutions, reflecting difficulty of the pumps to inject a larger volume of a viscous solution.

\section{Discussion}

This exploratory study suggests that relatively large SC injections (up to $3 \mathrm{~mL}$ ) are well tolerated, regardless of injection flow rate and fluid viscosity, when injected in the abdomen.

While there is widespread belief that the upper limit for a given $\mathrm{SC}$ injection is approximately $1 \mathrm{~mL}$ (driven by injection tolerance), ${ }^{22}$ these study results suggest that the upper limit for a given $\mathrm{SC}$ volume in the abdomen, merely based on injection tolerance, is well above $1 \mathrm{~mL}$. As demonstrated in this study, volumes up to $3 \mathrm{~mL}$ were well tolerated. Regardless of the relatively large volumes injected $(2-3 \mathrm{~mL})$, the overall tolerance of the SC injections was good. It is interesting to note that the mean injection pain measured using $100 \mathrm{~mm}$ VAS was $17.1 \mathrm{~mm}$ and was not clinically significantly higher (less than $10 \mathrm{~mm})^{23,24}$ than the mean pain recorded after needle insertion (VAS $12 \mathrm{~mm}$ ). This suggests that a major component of the overall SC injection pain originates from the needle insertion alone. In comparison to published studies that assessed SC injection pain, these results are lower; ${ }^{10,21}$ however, they are similar when limiting comparisons to pain assessed on abdominal injections only. ${ }^{21,25}$

Of the three parameters studied, solution viscosity was the factor with the higher impact on pain. Surprisingly, it was less painful with increased viscosity. This difference is statistically significant. To date, similar results have not been found published and therefore these results cannot be confirmed by other studies evaluating the influence of viscosity on injection pain.

The impact of volume injection on pain was not found to be statistically significant in this study, unlike in observations 
found in published studies ${ }^{21,22,26}$ where injection of 1 and $1.5 \mathrm{~mL}$ volumes caused significantly more pain than lower volumes (eg, 0.2 and $0.5 \mathrm{~mL}$ ). However, it may be that the differences observed across different volumes may be inconsequential when focusing on abdomen injections only. ${ }^{21}$ The results of this study in combination with those of Heise et $\mathrm{al}^{21}$ suggest that from a pain standpoint, volumes up to $3 \mathrm{~mL}$ (and probably higher) may be injected in the abdomen, while the same would likely be poorly tolerated (at least with fast rates) in the thigh. Therefore, these observations suggest that the abdomen should be the preferred injection site when there is a need to inject larger volumes in the SC space.

Although this study compared two flow rates that are 15-fold different, this parameter had no impact either on injection pain or on general tolerance. The same conclusion was made in the Heise et $\mathrm{al}^{21}$ study, where injection speeds from 0.15 to $0.45 \mathrm{~mL} / \mathrm{s}$ (including $0.30 \mathrm{~mL} / \mathrm{s}$ ) were evaluated, although the differences in compared flow rates were smaller. Therefore, these results suggest that the administration of $3 \mathrm{~mL}$ solutions subcutaneously in the abdomen at rates of up to $0.30 \mathrm{~mL} / \mathrm{s}$ is well tolerated.

In this study, the skin tolerance following the injections was acceptable although erythema was recorded in $72 \%$ of cases 15 minutes after injection. Results observed are lower than those recorded in other studies ${ }^{21,25,27}$ likely because the recorded reactions did not include bruising. Two (1.4\%) swelling/indurations were considered severe immediately after the injection, but one was moderate 15 minutes later. Both were observed in the $3 \mathrm{~mL}$-fast rate, resulting in a $16.6 \%$ rate for this subgroup; representing a significant difference. Therefore, skin tolerance may represent a limitation for frequent fast injections although they are well accepted from a pain injection standpoint. In the Herceptin (Genentech, South San Francisco, CA, USA) clinical studies (HannaH ${ }^{6}$ and $\operatorname{PrefHer}^{28}$ ), skin tolerance was not perceived as an issue at a $<0.02 \mathrm{~mL} / \mathrm{s}$ flow rate, which is consistent with our results. Patients preferred SC to IV route despite the increased number of grade 1 adverse events ${ }^{6,28}$ suggesting that skin tolerance inconveniences are outweighed by the convenience of the SC administration. However, Herceptin formulation has recombinant hyaluronidase excipient, ${ }^{29}$ which is used to increase permeability of connective tissue. ${ }^{3}$

The localization of the injected fluid, assessed by echography, indicated that the solution was exclusively in the SC tissue in $92 \%$ of cases. This ratio did not change significantly across injection types. The injected fluid location was deeper with $3 \mathrm{~mL}$ injections (as expected); it was also the case with increasingly viscous solutions.
Actual volume injected corresponded to $83 \%$ of the target. This ratio was moderately higher for $3 \mathrm{~mL}$ and $0.30 \mathrm{~mL} / \mathrm{s}$ injections, ruling out the hypothesis that this low injected volume ratio could be related to difficulty of the SC tissue to accommodate the solution (increased back pressure). Rather, the electric pumps used in this study may have contributed to this observation, as they are designed to be used for several hours for injection of nonviscous fluids; their validation is based on the volume administered after 1 hour, and several minutes are needed for the pump to reach the target flow rate, the ratio at start of infusion being slower than programmed. Actually, a stabilization period of 45-90 minutes may be required for this type of electric pump system. ${ }^{30}$ In our study, the injection lasted less than 5 minutes; therefore, the $83 \%$ injection volume is logical, to a certain extent. Nevertheless, because the injected volume ratio is homogeneously distributed across flow rate and different volume injections, we do not think this slightly lower volume injected ratio bias impacts the study conclusions: the implications are that actual maximum volumes and flow rates tested are $2.5 \mathrm{~mL}$ and 0.75 $\mathrm{mL} / \mathrm{s}$, respectively, instead of $3 \mathrm{~mL}$ and $0.9 \mathrm{~mL} / \mathrm{s}$.

The injected volume ratio significantly decreased with increasing fluid viscosity. Injection system occlusions were recorded in $15 \%$ (22) of cases resulting in difficulties to complete the injection for the last $20 \%$ of the fluid. This strongly indicates that the injection delivery system required for fast and large volumes of viscous injections needs to be specifically designed, independent of the subject's tolerance. Indeed, this problem was not identified during the prestudy pump selection tests. As it was essential for the study design that injections were performed with the same pump, it was not possible to correct the issue when it was identified. It is difficult to evaluate whether this phenomenon impacts the study conclusion, indicating that high-viscosity fluids are less painful on injection. On one hand, the volume actually delivered was lower with viscous fluid injections, but on the other hand, the injected volume did not have an effect on injection pain. Moreover, the complementary analyses conducted on the 122 complete injections were found to confirm the results: viscosity effect remained almost significant $(P=0.08)$.

There are several limitations of the study. As previously discussed, the pump performance when injecting viscous solutions seems to bias the results recorded at least with respect to flow rate and volume tolerance. A second limitation resides in the characteristic of the injected solutions. Pain on injection varies with solution characteristics (eg, $\mathrm{pH}$, tonicity, osmolarity, fluid temperature, drug structure, concentration, and the type of excipients used). ${ }^{31}$ As a result, 
it is possible that the descriptive part of the study conclusions, especially tolerance, would not directly apply to different formulations. Nevertheless, the observed comparisons across volumes, flow rates, and viscosities are useful in ruling them out as major contributors to injection tolerance (as defined by pain). Nevertheless, observations made with this neutral solution may represent a good basis when testing actual SC formulations.

\section{Conclusion}

In summary, the results of this study suggest that solutions of up to $3 \mathrm{~mL}$ (or at least $2.5 \mathrm{~mL}$ considering the pump limitations), and up to $15-20 \mathrm{cP}$ can be injected into the abdomen within 10 seconds without pain tolerance concerns. This observation, based on the flow rate representative of an autoinjector for self-injection, opens the path to a generation of larger volume autoinjectors. The example of trastuzumab shows that the SC administration of monoclonal antibodies, instead of IV, simplifies long-term oncology therapy for patients, improves their quality of life, saves medical personnel time, brings flexibility in treatment administration, and is preferred to conventional IV infusion. ${ }^{14,28}$

Significant rates of severe indurations have been observed when injecting subcutaneously a 15-20 cP solution at $0.30 \mathrm{~mL} / \mathrm{s}$ flow rate, but the indurations rapidly recovered. This may be a limitation for frequent (eg, daily) injections for which, according to our results, a lower flow rate could be preferred. This would result in longer injections to ensure that the whole dose has been administered. For weekly or less frequent injections, which is usually the case with monoclonal antibodies, this rapidly recovered swelling/induration risk needs to be balanced with the benefit and comfort of a shorter injection time.

With the $0.02 \mathrm{~mL} / \mathrm{s}$ flow rate, no limitation was recorded suggesting that even a high-viscosity, large-volume solution is likely to be well accepted. This is confirmed by the HannaH study results where it was observed that $5 \mathrm{~mL}$ of trastuzumab could be administered subcutaneously within 5 minutes with an acceptable tolerance in the thigh. ${ }^{6}$

Study results also suggest that injection pain decreases with the viscosity of the fluid injected. To our knowledge, this is the first time the impact of viscosity on pain has been evaluated. This result should be confirmed by additional studies using devices specifically adapted for injection of viscous solutions. The confirmation of these results could open the door to support more self-administration of viscous biological molecules.

\section{Acknowledgments}

The authors thank Dr Marie Steidel for performing and interpreting the ultrasound examinations, the clinical investigation staff of Eurofins OPTIMED Clinical Research for their help in conducting the clinical study, and Dr Luc Marticou (BD Medical Pharmaceutical Systems) for his writing assistance for this manuscript.

\section{Disclosure}

The study was funded and sponsored by Eli Lilly and Company. CB, OFS, CG, and FS are employees of BD Medical Pharmaceutical Systems. TW and HER are employees of Eli Lilly and Company. The authors report no other conflicts of interest in this work.

\section{References}

1. Dougan M, Dranoff G. Immune therapy for cancer. Annu Rev Immunol. 2009;27:83-117.

2. Rader RA. FDA Biopharmaceutical Product Approvals and Trends in 2012. Westborough, MA: BioProcess International; 2011.

3. Jackisch C, Muller V, Maintz C, Hell S, Ataseven B. Subcutaneous administration of monoclonal antibodies in oncology. Geburtshilfe Frauenheilkd. 2014;74(4):343-349.

4. Kling J. Highly Concentrated Protein Formulations. Westborough, MA: BioProcess International; 2014.

5. Leveque D. Subcutaneous administration of anticancer agents. Anticancer Res. 2014;34(4):1579-1586.

6. Ismael G, Hegg R, Muehlbauer S, et al. Subcutaneous versus intravenous administration of (neo)adjuvant trastuzumab in patients with HER2-positive, clinical stage I-III breast cancer (HannaH study): a phase 3, open-label, multicentre, randomised trial. Lancet Oncol. 2012;13(9):869-878.

7. McCormack PL. Omalizumab: a review of its use in patients with chronic spontaneous urticaria. Drugs. 2014;74(14):1693-1699.

8. Borah BJ, Huang X, Zarotsky V, Globe D. Trends in RA patients' adherence to subcutaneous anti-TNF therapies and costs. Curr Med Res Opin. 2009;25(6):1365-1377.

9. Khraishi MM. Experience with subcutaneous abatacept for rheumatoid arthritis: an update for clinicians. Ther Adv Musculoskelet Dis. 2014; 6(5):159-168.

10. Kivitz A, Cohen S, Dowd JE, et al. Clinical assessment of pain, tolerability, and preference of an autoinjection pen versus a prefilled syringe for patient self-administration of the fully human, monoclonal antibody adalimumab: the TOUCH trial. Clin Ther. 2006;28(10):1619-1629.

11. Scott LJ. Etanercept: a review of its use in autoimmune inflammatory diseases. Drugs. 2014;74(12):1379-1410.

12. Sohn W, Simiens MA, Jaeger K, Hutton S, Jang G. The pharmacokinetics and pharmacodynamics of denosumab in patients with advanced solid tumours and bone metastases: a systematic review. $\mathrm{Br} J \mathrm{Clin}$ Pharmacol. 2014;78(3):477-487.

13. Melichar B, Studentova H, Kalabova H, Vitaskova D. Role of subcutaneous formulation of trastuzumab in the treatment of patients with HER2-positive breast cancer. Immunotherapy. 2014;6(7):811-819.

14. Pivot X, Gligorov J, Muller V, et al. Patients' preferences for subcutaneous trastuzumab versus conventional intravenous infusion for the adjuvant treatment of HER2-positive early breast cancer: final analysis of 488 patients in the international, randomized, two-cohort PrefHer study. Ann Oncol. 2014;25(10):1979-1987.

15. Jezek J, Rides M, Derham B, et al. Viscosity of concentrated therapeutic protein compositions. Adv Drug Deliv Rev. 2011;63(13):1107-1117. 
16. Liu J, Nguyen MD, Andya JD, Shire SJ. Reversible self-association increases the viscosity of a concentrated monoclonal antibody in aqueous solution. J Pharm Sci. 2005;94(9):1928-1940.

17. Narasimhan C, Mach H, Shameem M. High-dose monoclonal antibodies via the subcutaneous route: challenges and technical solutions, an industry perspective. Ther Deliv. 2012;3(7):889-900.

18. Saluja A, Kalonia DS. Nature and consequences of protein-protein interactions in high protein concentration solutions. Int J Pharm. 2008; 358(1-2):1-15.

19. Dias C, Abosaleem B, Crispino C, Gao B, Shaywitz A. Tolerability of high-volume subcutaneous injections of a viscous placebo buffer: a randomized, crossover study in healthy subjects. AAPS PharmSciTech. Epub 2015 Feb 19.

20. US Army Medical Department. Distance Learning Course Lesson 2: administer an intramuscular injection. Available from: http:// brooksidepress.org/Products/Administer_IM_SQ_and_ID_Injections/ lesson_2_Section_1.htm. Accessed September 8, 2015.

21. Heise T, Nosek L, Dellweg S, et al. Impact of injection speed and volume on perceived pain during subcutaneous injections into the abdomen and thigh: a single-centre, randomized controlled trial. Diabetes Obes Metab. 2014;16(10):971-976.

22. Jorgensen JT, Romsing J, Rasmussen M, Moller-Sonnergaard J, Vang L, Musaeus L. Pain assessment of subcutaneous injections. Ann Pharmacother. 1996;30(7-8):729-732.

23. Campbell WI, Patterson CC. Quantifying meaningful changes in pain. Anaesthesia. 1998;53(2):121-125.
24. Todd KH. Clinical versus statistical significance in the assessment of pain relief. Ann Emerg Med. 1996;27(4):439-441.

25. Chan H. Effects of injection duration on site-pain intensity and bruising associated with subcutaneous heparin. J Adv Nurs. 2001; 35(6):882-892.

26. Berteau C, Schwarzenbach F, Donazzolo Y, et al. Evaluation of performance, safety, subject acceptance, and compliance of a disposable autoinjector for subcutaneous injections in healthy volunteers. Patient Prefer Adherence. 2010;4:379-388.

27. Pourghaznein T, Azimi AV, Jafarabadi MA. The effect of injection duration and injection site on pain and bruising of subcutaneous injection of heparin. J Clin Nurs. 2014;23(7-8):1105-1113.

28. Pivot X, Gligorov J, Muller V, et al. Preference for subcutaneous or intravenous administration of trastuzumab in patients with HER2positive early breast cancer (PrefHer): an open-label randomised study. Lancet Oncol. 2013;14(10):962-970.

29. European Medicines Agency. CHMP Assessment Report-Trastuzumab (Herceptin). London, UK; European Medicines Agency; 2013.

30. European Standard. BS EN 60601-2-24:1998. Medical Electrical Equipment-Part 2: Particular Requirements for the Safety of Infusion Pumps and Controllers. London, UK: British Standards Institution; 1998.

31. Brazeau GA, Cooper B, Svetic KA, Smith CL, Gupta P. Current perspectives on pain upon injection of drugs. J Pharm Sci. 1998;87(6): $667-677$. 


\section{Supplementary material}

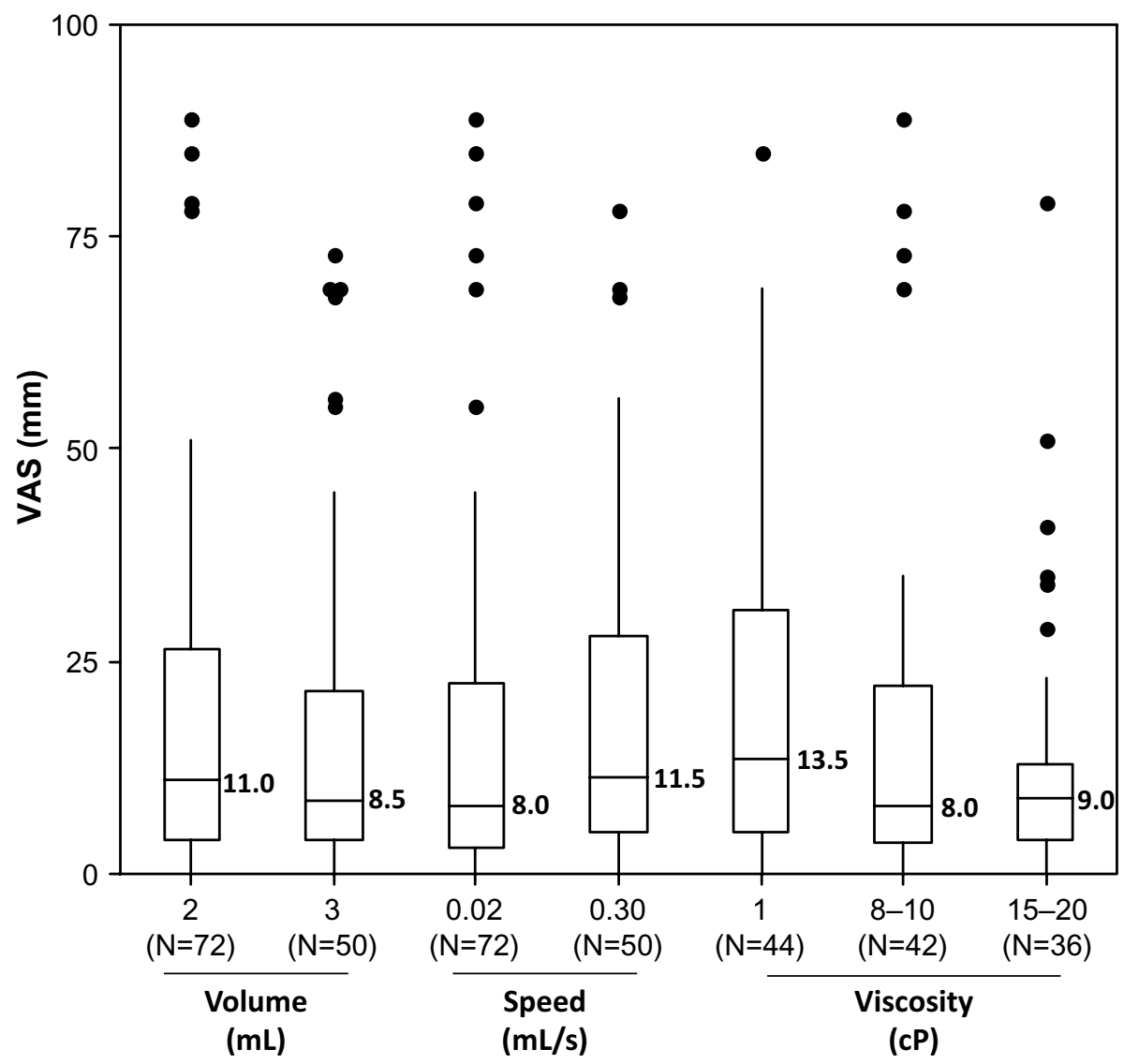

Figure SI Perceived injection pain after the end of injection for the 122 complete injections.

Notes: VAS was used to assess pain and ranges from $0 \mathrm{~mm} /$ no pain to $100 \mathrm{~mm} /$ extreme pain; medians are indicated. Small and not significant difference is observed between low and high viscosity injection $(P=0.08)$.

Abbreviation: VAS, visual analog scale.

\section{Publish your work in this journal}

Medical Devices: Evidence and Research is an international, peerreviewed, open access journal that focuses on the evidence, technology, research, and expert opinion supporting the use and application of medical devices in the diagnosis, treatment and management of clinical conditions and physiological processes. The identification of novel devices and optimal use of existing devices which will lead to improved clinical outcomes and more effective patient management and safety is a key feature. The manuscript management system is completely online and includes a quick and fair peer-review system. Visit http://www. dovepress.com/testimonials.php to read real quotes from authors. 